SNUTP-98-012

\title{
Quantum Inflaton Dynamics
}

\author{
Dongsu Bak, ${ }^{a *}$ Sang Pyo Kim ${ }^{b \dagger}$, Sung Ku Kim, ${ }^{c \ddagger}$, Kwang-Sup Soh, ${ }^{d \S}$, and Jae Hyung \\ Yee $^{e * *}$ \\ ${ }^{a}$ Department of Physics, University of Seoul, Seoul 130-743, Korea \\ ${ }^{b}$ Department of Physics, Kunsan National University, Kunsan 573-701, Korea \\ ${ }^{c}$ Department of Physics, Ewha Womans University, Seoul 120-750, Korea \\ ${ }^{d}$ Department of Physics Education, Seoul National University, Seoul 151-742, Korea \\ e Department of Physics, Yonsei University, Seoul 120-749, Korea
}

(March 20, 2018)

\begin{abstract}
We show that the quantum dynamics of a real scalar field for a large class of potentials in the symmetric Gaussian state, where the nonperturbative quantum contributions are taken into account, can be described equivalently by a two-dimensional nonlinear dynamical system with a definite angular momentum (U(1) charge of a complex theory). It is found that the Gaussian state with a nearly minimal uncertainty and a large quantum fluctuation, as an initial condition, naturally explains the most of the essential features of the early stage of the inflationary Universe.
\end{abstract}

PACS number(s): 98.80.Cq; 04.62.+v

\footnotetext{
*Electronic address: dsbak@mach.scu.ac.kr

†Electronic address: sangkim@knusun1.kunsan.ac.kr

$\ddagger$ Electronic address: skkim@theory.ewha.ac.kr

§Electronic address: kssoh@phya.snu.ac.kr

**Electronic address: jhyee@phya.yonsei.ac.kr
} 
The standard cosmological model combined with particle physics at high energy (temperature) is plagued with the monopole, the horizon, the flatness problems, and so on. A major breakthrough to solve most of these problems was found in the inflation paradigm invented by Guth [1]. (For review and references, see [2].) His simple but attractive idea, the so-called old inflation scenario, is that an inflaton (homogeneous scalar field) undergoes a first order phase transition from a symmetric vacuum as the Universe expands and thereby the temperature drops, and the energy density of inflaton captured in the false vacuum drives an exponential expansion of the de Sitter phase. However, this scenario has a major defect, the graceful exit problem [3]. The new inflation scenario [4] based on a second order phase transition overcomes the graceful exit problem, but raises another problem involving the fine tuning of coupling constants. It is also possible to solve the graceful exit problem in the context of Jordan-Brans-Dicke theory [5]. The chaotic inflation scenario introduced by Linde [6] does not make use of phase transitions but rather investigate various initial distributions of the inflaton that lead to inflation. Furthermore, this scenario is model-independent in the sense that one can obtain a significant inflation for a large class of potentials.

All these scenarios are based on the classical gravity of the Friedmann equation and the scalar field equation on the Friedmann-Robertson-Walker (FRW) universe, assuming its validity even at the very early Universe. However, quantum effects of matter fields such as quantum fluctuations are expected to play a significant role in this regime, though quantum gravity effects are still negligible. The proper description of cosmological model should be in terms of the semiclassical gravity of the semiclassical Friedmann equation with quantized matter fields as far as inflation is concerned.

In this Letter, we pursue the quantum dynamics of a real-valued inflaton (homogeneous real scalar field) for a large class of potentials in the FRW Universe. In the Schrödinger picture, the scalar inflaton theory is approximately but quite accurately described by a symmetric Gaussian state for an extremal configuration of a bosonic condensate of scalar particles. The symmetric Gaussian state can be explicitly found by using a nonperturbative method of Ref. [7] or a direct Gaussian wave function as of Refs. [8,9]. The resulting equations are two-dimensional coupled nonlinear equations where the dynamical variables are the dispersion of inflaton and the scale factor of the FRW Universe.

We shall begin with a spatially flat FRW metrid

$$
d s^{2}=-d t^{2}+a^{2}(t) d \mathbf{x}^{2},
$$

and consider a real homogeneous and isotropic inflaton described by the Hamiltonian

$$
H(t)=\frac{p^{2}}{2 a^{3}(t)}+a^{3}(t)\left(\frac{m^{2}}{2} \phi^{2}+\frac{m_{P}^{4} \lambda_{2 n}}{(2 n)}\left(\frac{\phi}{m_{P}}\right)^{2 n}\right),
$$

where $p=a^{3} \dot{\phi}$ is the momentum conjugate to the real inflaton field. The classical equation of motion for the inflaton field reads

\footnotetext{
${ }^{1}$ We shall use units system $c=\hbar=1$ and $m_{P}^{2}=\frac{1}{G}$ and follow the notations in Ref. [6], in which the Planck length and time are $l_{P}=t_{P}=\frac{1}{m_{P}}, \phi$ and $a$ have the dimension $m_{P}$ and $\frac{1}{m_{P}}$, respectively, and $\lambda_{2 n}$ is dimensionless.
} 


$$
\ddot{\phi}+3\left(\frac{\dot{a}}{a}\right) \dot{\phi}+m^{2} \phi+m_{P}^{2} \lambda_{2 n}\left(\frac{\phi}{m_{P}}\right)^{2 n-2} \phi=0
$$

while the the scale factor $a(t)$ is governed by the Friedmann equation

$$
\left(\frac{\dot{a}(t)}{a(t)}\right)^{2}=\frac{8 \pi}{3 a^{3} m_{P}^{2}} H(t) .
$$

In this classical context, it is known that the inflation may indeed occur but the unnatural fine tuning of the initial data is, more or less, needed as will be seen. But before dealing with the classical gravity, we shall first analyze the quantum theory and obtain the classical theory as its limit.

Upon quantization of the real inflaton, the Schrödinger equation for quantum field

$$
i \frac{\partial}{\partial t} \Psi(\phi, t)=\hat{H}(t) \Psi(\phi, t),
$$

determines the time evolution of the inflaton, whereas the Friedmann equation at its semiclassical level

$$
\left(\frac{\dot{a}(t)}{a(t)}\right)^{2}=\frac{8 \pi}{3 a^{3} m_{P}^{2}}\langle\hat{H}\rangle
$$

describes the evolution of the Universe.

There is a rather standard method to find an approximate Gaussian state that extremizes the energy (see e.g. Refs. [7 9]). Although one may use a more generic Gaussian wave function, we shall use a symmetric Gaussian state [8]

$$
\Psi(\phi, t)=\frac{1}{\left(2 \pi \chi^{2}(t)\right)^{1 / 4}} \exp \left[-\left(\frac{1}{4 \chi^{2}(t)}-i \frac{\pi_{\chi}(t)}{2 \chi(t)}\right) \phi^{2}\right]
$$

as our trial state. The real functions $\chi$ and $\pi_{\chi}$ are time-dependent parameters, whose time-dependence will be determined below by the Dirac action principle. We are going to extremize the effective action

$$
I_{\mathrm{eff}}=\int d t\left\langle\Psi\left|\left[i \partial_{t}-\hat{\mathbf{H}}(t)\right]\right| \Psi\right\rangle
$$

which is the Dirac action except for its wave function is now limited by the Gaussian form in Eq. (7). By a straightforward computation, one finds that the effective action is given by

$$
I_{\mathrm{eff}}=\int d t\left[\pi_{\chi} \dot{\chi}-H_{\mathrm{eff}}\left(\pi_{\chi}, \chi\right)\right]
$$

where

$$
H_{\mathrm{eff}}\left(\pi_{\chi}, \chi\right)=\frac{\pi_{\chi}^{2}}{2 a^{3}}+a^{3} V_{\mathrm{eff}}(\chi)
$$

with the effective potential,

$$
V_{\mathrm{eff}}(\chi)=\frac{1}{8 a^{6} \chi^{2}}+\frac{m^{2}}{2} \chi^{2}+\frac{m_{P}^{4} \lambda_{2 n}^{Q}}{(2 n)}\left(\frac{\chi}{m_{P}}\right)^{2 n},
$$


where $\lambda_{2 n}^{Q}=\frac{(2 n) !}{2^{n} n !} \lambda_{2 n}$ is an effective coupling constant. Upon extremization of the effective action (9), one finds that our system is described by the Hamilton's equations

$$
\begin{aligned}
a^{3} \dot{\chi} & =\pi_{\chi} \\
\dot{\pi}_{\chi} & =-a^{3} \frac{\partial}{\partial \chi} V_{\text {eff }}(\chi) \\
& =\frac{1}{4 a^{3} \chi^{3}}-a^{3}\left(m^{2} \chi+m_{P}^{3} \lambda_{2 n}^{Q}\left(\frac{\chi}{m_{P}}\right)^{2 n-1}\right)
\end{aligned}
$$

The semiclassical Friedmann equation becomes

$$
\left(\frac{\dot{a}(t)}{a(t)}\right)^{2}=\frac{8 \pi}{3 a^{3} m_{P}^{2}} H_{\mathrm{eff}}
$$

One can also understand the physics of the above system from a different point of view using the method of Ref. [7], which is based on the technique of solving the Schrödinger equation for the time-dependent Hamiltonian system [10]. One introduces the annihilation operator of a Fock space [1] redefined as dimensionless quantity,

$$
\hat{A}=\frac{\varphi^{*}(t)}{m_{P}} \hat{p}-m_{P} a^{3}(t) \dot{\varphi}^{*}(t) \hat{\phi}, \hat{A}^{\dagger}=\text { h.c, }
$$

such that

$$
\left[\hat{A}, \hat{A}^{\dagger}\right]=i a^{3}\left(\varphi^{*} \dot{\varphi}-\dot{\varphi}^{*} \varphi\right)=1
$$

and $\varphi(t)$ in Eq. (14) is a complex scalar variable with the same dimension as the classical field $\phi$. One then expands the Hamiltonian in terms of $\hat{A}$ and $\hat{A}^{\dagger}$, and truncates it up to the quadratic terms $\hat{H}_{(2)}$ for an approximation. The requirement that $\hat{A}$ and $\hat{A}^{\dagger}$ should be the solutions of the Liouville-Neumann equation,

$$
i \frac{\partial \hat{A}}{\partial t}+\left[\hat{A}, \hat{H}_{(2)}\right]=0
$$

leads to the equation of motion for the $\varphi$-field

$$
\ddot{\varphi}+3\left(\frac{\dot{a}}{a}\right) \dot{\varphi}+m^{2} \varphi+m_{P}^{2} \lambda_{2 n}^{Q}\left(\frac{\varphi^{*} \varphi}{m_{P}^{2}}\right)^{n-1} \varphi=0 .
$$

If one sets $\varphi=\chi e^{-i \theta}$, Eq. (17) reduces to Eq. (12) with the constraint (15) expressed as

$$
Q \equiv 2 a^{3} \chi^{2} \dot{\theta}=1
$$

We have thus found that the quantum dynamics of the real inflaton theory is equivalently described by the two-dimensional nonlinear dynamical system. The expectation value of the Hamiltonian gives rise to the effective Hamiltonian

$$
H_{\mathrm{eff}}(t)=\langle\hat{H}(t)\rangle=a^{3}\left\{\frac{1}{2} \dot{\varphi}^{*} \dot{\varphi}+\frac{m^{2}}{2} \varphi^{*} \varphi+\frac{m_{P}^{4} \lambda_{2 n}}{(2 n)}\left(\frac{\varphi^{*} \varphi}{m_{P}^{2}}\right)^{n}\right\} .
$$


It should be noted that this complex system has a $U(1)$ symmetry under the transformation $\varphi \rightarrow e^{i \alpha} \varphi$, and that the commutation relation $\left[\hat{A}, \hat{A}^{\dagger}\right]=1$ indeed determines the charge to be unity. In this framework one can easily obtain the higher order corrections to the Gaussian state (7) ?2.

A few comments are in order. First, we note that the effective potential (11) comes from the nonperturbative quantum contributions and the factor $(2 n) ! /\left(2^{n} n !\right)$ of $\lambda_{2 n}^{Q}$ in Eqs. (12), (17) and (13), actually accounts for the number of symmetric loop diagrams from the higher order self-interactions and, hence, enhances the effective coupling constants in the very early Universe when the quantum effects of matter fields are expected to be important. For the massive real inflaton without higher order interaction (i.e. $\lambda_{2 n}=0$ ), the wave function (7) determined in this way is indeed the exact state of Eq. (5), and the other excited quantum states can be constructed by acting the creation operators (14) [11]. Once the higher order coupling is turned on, the Gaussian state is no longer exact, but it is also known that it describes quite accurately the system even for a strong coupling constant.

We are now able to find the two asymptotic solutions to Eq. (12). First, we consider the quantum dynamics at the very early Universe. Eq. (12) can be written as a second order equation

$$
\ddot{\chi}+3\left(\frac{\dot{a}}{a}\right) \dot{\chi}-\frac{1}{4 a^{6} \chi^{3}}+m^{2} \chi+m_{P}^{3} \lambda_{2 n}^{Q}\left(\frac{\chi}{m_{P}}\right)^{2 n-1}=0 .
$$

The symmetric Gaussian state (đ) for the inflaton has the dispersions

$$
\begin{aligned}
& \Delta \phi=\sqrt{\left\langle\hat{\phi}^{2}\right\rangle-\langle\hat{\phi}\rangle^{2}}=\chi, \\
& \Delta \pi=\sqrt{\left\langle\hat{\pi}^{2}\right\rangle-\langle\hat{\pi}\rangle^{2}}=a^{3}\left(\dot{\chi}^{2}+\chi^{2} \dot{\chi}^{2}\right)^{1 / 2}
\end{aligned}
$$

where $\langle\hat{\pi}\rangle=\langle\hat{\phi}\rangle=0$. The uncertainty relation becomes

$$
\Delta \phi \Delta \pi=\frac{1}{2}\left(1+4 \pi_{\chi}^{2} \chi^{2}\right)^{1 / 2}
$$

where we used Eq. (18). A nearly minimal uncertainty can be achieved when $\pi_{\chi} \chi \sim 0$, that is, either $\pi_{\chi} \sim 0$ or $\chi \sim 0$. We propose an inflaton's initial quantum state with $\pi_{\chi} \sim 0$ and a large quantum fluctuation $\chi>\chi_{c}$, where

$$
\frac{\chi_{c}}{m_{P}}=\min \cdot\left\{\left[\frac{1}{\lambda_{2 n}^{Q}}\left(\frac{m}{m_{P}}\right)^{2}\right]^{1 /(2 n-2)},\left[\frac{1}{4 \lambda_{2 n}^{Q}}\left(\frac{m}{a_{0}}\right)^{6}\right]^{1 /(2 n+2)}\right\} .
$$

Under this initial condition the first term and the centrifugal potential term in Eq. (20) are small compared with the other two terms, and Eq. (20) reduces to the equation for classical inflaton when the dispersion $\chi$ is interpreted as $\phi$. For this large quantum fluctuation, Eq. (20) may be approximated as

\footnotetext{
${ }^{2}$ For the systematic improvement of the approximation, see Ref. [7] and the detailed deviations from the exact results in this Gaussian approximation, is dealt with in Ref. [9].
} 


$$
3\left(\frac{\dot{a}}{a}\right) \dot{\chi}+m_{P}^{3} \lambda_{2 n}^{Q}\left(\frac{\chi}{m_{P}}\right)^{2 n-1} \simeq 0
$$

Since the kinetic term $\frac{\pi_{\chi}^{2}}{2 a^{3}}$ in $H_{\text {eff }}$ is much smaller than $V_{\text {eff }}$ for large $\chi$, by substituting an approximate Friedmann equation

$$
\frac{\dot{a}}{a} \simeq \sqrt{\frac{4 \pi m_{P}^{2} \lambda_{2 n}^{Q}}{3 n}}\left(\frac{\chi}{m_{P}}\right)^{n},
$$

into Eq. (24), we obtain the solutions

$$
\begin{aligned}
& \chi(t) \simeq \chi\left(t_{i}\right) \exp \left(-\sqrt{\frac{m_{P}^{2} \lambda_{2 n}^{Q}}{6 \pi}}\left(t-t_{i}\right)\right),(n=2), \\
& \chi(t) \simeq \chi\left(t_{i}\right)\left[1+\sqrt{\frac{n(n+2)^{2} m_{P}^{2} \lambda_{2 n}^{Q}}{12 \pi}}\left(t-t_{i}\right)\right]^{1 /(n-2)},(n \geq 3) .
\end{aligned}
$$

In all cases of $n$ one has a period of inflation described by

$$
a(t) \simeq a\left(t_{i}\right) \exp \left[\sqrt{\frac{4 \pi m_{P}^{2} \lambda_{2 n}^{Q}}{3 n}} \int_{t_{i}}^{t}\left(\frac{\chi}{m_{P}}\right)^{n}(t) d t\right],
$$

that follows from the Friedmann equation. These solutions are the same as those of the chaotic inflation model [2, 6]. The big difference, however, is that in quantum dynamical model of inflation the large quantum fluctuation with the nearly minimal uncertainty and the non-perturbative quantum contributions at the very early Universe does drive the quasiexponential expansion of the Universe. As the Universe inflates, $\Delta \phi$ decreases but

$\Delta \pi_{\chi}$ grows and the symmetric Gaussian state becomes sharply peaked, showing classical features.

Second, we consider the late evolution of the Universe. Using nonlinear system theory [12], we find a limit cycle when $\frac{\partial}{\partial \chi} V_{\text {eff }}(\chi)=0$ :

$$
\frac{1}{4 a^{6} \chi^{4}}=m^{2}+m_{P}^{2} \lambda_{2 n}^{Q}\left(\frac{\chi}{m_{P}}\right)^{2(n-1)}
$$

As the Universe expands, the quantum fluctuation $\chi$ decreases due to the friction from Hubble parameter and behaves as a classical inflaton field. The equilibrium is determined dominantly by the mass term, and the motion of the effective inflaton tends toward the limit cycle of a coherent oscillation constrained approximately by

$$
\chi^{2} a^{3} \simeq \frac{1}{2 m}
$$

The Friedmann equation along the limit cycle of the motion leads to a power-law expansion

$$
a(t) \simeq\left(\frac{3 \pi m}{2 m_{P}^{2}}\left(1+\frac{1}{n}\right) t^{2}\right)^{1 / 3}
$$

which is valid for the later time. 
Finally, we extend the potential in (目) to be an arbitrary analytic and bounded potential $V(\phi)=F(\phi)$, which is symmetric, $F(-x)=F(x)$. One may then expand the potential in Taylor series by

$$
F(\phi)=\sum_{n=1} \frac{m_{P}^{4} C_{2 n}}{(2 n) !}\left(\frac{\phi}{m_{P}}\right)^{2 n},
$$

where the vacuum energy is adjusted to zero. By the same analysis as before, one may obtain the effective potential from quantum fluctuation

$$
V_{\mathrm{eff}}=\frac{1}{8 a^{6} \chi^{2}}+\sum_{n=1} \frac{m_{P}^{4} C_{2 n}}{2^{n} n !}\left(\frac{\chi^{2}}{m_{P}^{2}}\right)^{n} .
$$

Now, most of the above analysis of the nonlinear system holds with a modification that the lowest term $n_{\min }$ plays the role of determining the limit cycle and the largest term $n_{\max }$ is driving an inflation.

In summary, we have studied the quantum dynamics of a real inflaton in its symmetric Gaussian state for a large class of potentials. We have found an equivalent two-dimensional nonlinear dynamical system for the dispersion of the inflaton and the conserved $\mathrm{U}(1)$ charge as an angular momentum. We have shown that the large quantum fluctuation of the inflaton with a minimal uncertainty naturally explains the early stage of the inflationary Universe. It was shown that the U(1) charge is fixed to a definite value, which plays the role of angular momentum in the two-dimensional nonlinear system. This is contrasted to the usual complex inflaton theory used in the inflation model [13, 14 or the wormhole model [15] since there is no a priori reason to take the $\mathrm{U}(1)$ charge to be fixed in these models.

We conclude with some comments that we have not dealt in detail with the enhancement of effective coupling constants and with the inhomogeneous degrees of freedom (i.e. the non-zero modes) in the real scalar field. Analysis on the non-zero modes may be interesting since they give rise to the density perturbation necessary for structure formation and may affect the inflation and the quantum dynamics of the real inflaton. The quantization of this interacting theory of the non-zero modes and the role of effective coupling constants in density perturbation require a further study.

This work was supported in parts by Center for Theoretical Physics, Seoul National University, and by the Basic Science Research Institute Program, Korea Ministry of Education under Project. No. BSRI-97-2418, BSRI-97-2425 and BSRI-97-2427. SPK was also supported by Non Directed Research Fund, Korea Research Foundation, 1997, and JHY by KOSEF Grant No. 97-07-02-02-01-3. 


\section{REFERENCES}

[1] A. H. Guth, Phys. Rev. D 23, 347 (1981).

[2] A. D. Linde, Particle Physics and Inflationary Cosmology (Harwood, Chur, Switzerland, 1990).

[3] S. W. Hawking, I. G. Moss, and J. M. Stewart, Phys. Rev. D 26, 2681 (1982); A. H. Guth and E. Weinberg, Nucl. Phys. B212, 321 (1983).

[4] A. D. Linde, Phys. Lett. 108B, 389 (1982); ibid. 114B, 431 (1982); A. Albrecht and P. J. Steinhardt, Phys. Rev. Lett. 48, 1220 (1982).

[5] D. La and P. J. Steinhardt, Phys. Rev. Lett. 62, 376 (1989).

[6] A. D. Linde, Phys. Lett. 129B 177 (1983).

[7] S. P. Kim, "Mean-Field Approach to Quantum Duffing Oscillator", physics/9702018 (unpublished); "Liouville-Neumann Approach to Nonperturbative Quantum Field Theory", to appear in Recent Developments in Nonperturbative Quantum Field Theory, the Proceedings of APCTP-ICTP Joint International Conference, Seoul 1997, hepth/9706052 (1997).

[8] O. Eboli, S.-Y. Pi, and M. Samiullah, Ann. Phys. 193, 102 (1989).

[9] G. H. Lee and J. H. Yee, Phys. Rev. D 56, 6573 (1997).

[10] H. R. Lewis, Jr. and W. B. Riesenfeld, J. Math. Phys. (N.Y.) 10, 1458 (1969).

[11] S. P. Kim, Class. Quantum Grav. 13, 1377 (1996); Phys. Rev. D 55, 7511 (1997); S. P. Kim, J.-Y. Ji, H.-S. Shin, and K.-S. Soh, Phys. Rev. D 56, 3756 (1997); S. P. Kim and D. N. Page, "Inflation in Semiclassical Gravity", SNUTP-97-126 (1997).

[12] S. Wiggins, Introduction to Applied Nonlinear Dynamical Systems and Chaos (SpringerVerlag, New York, 1990).

[13] I. M. Khalatnikov and A. Mezhlumian, Phys. Lett. A169, 308 (1992); I. M. Khalatnikov and P. Schiller, Phys. Lett. B302, 176 (1993); L. Amendola, I. M. Khalatnikov, M. Litterio, and F. Occhionero, Phys. Rev. D 49, 1881 (1994); A. Y. Kamenshchik, I. M. Khalatnikov, and A. V. Toporensky, "Simplest cosmological model with the scalar field", gr-qc/9801064.

[14] D. Scialom and P. Jetzer, Phys. Rev. D 51, 5698 (1995).

[15] K. Lee, Phys. Rev. Lett. 61, 263 (1988); Phys. Rev. D 50, 5333 (1994). 